\title{
Definición del KPi Porcentaje de sacos con defecto y Takt Time
}

\section{Definition of KPi Percentage of bags with defect and Takt Time}

HERNÁNDEZ-PASTRANA, Verónica Petra*, KIDO-MIRANDA, Juan Carlos, PÉREZ-CABRERA, Pascual Felipe, y RODRÍGUEZ-BUCIO, Norma

Instituto Tecnológico de Iguala / Tecnológico Nacional de México

ID $1^{\text {er }}$ Autor: Verónica Petra, Hernández-Pastrana / ORC ID: 0000-0002-1768-0220, Researcher ID Thomson: X-38652018, CVU CONACYT ID: 550871

ID $1^{\text {er }}$ Coautor: Juan Carlos, Kido-Miranda / ORC ID: 0000-003-3375-627X, Researcher ID Thomson: X-3884-2018, CVU CONACYT ID: 370488

ID $2^{\text {do }}$ Coautor: Pascual Felipe, Pérez-Cabrera / ORC ID: 0000-0002-5432-6730, Researcher ID Thomson: X-3905-2018, CVU CONACYT ID: 953461

ID $3^{\text {er }}$ Coautor: Norma, Rodríguez-Bucio / ORC ID: 0000-0001-5287-0946, Researcher ID Thomson: X-3895-2018, CVU CONACYT ID: 550895

DOI: $10.35429 /$ JTO.2019.11.3.21.29

Recibido 8 de Junio, 2019, Aceptado, 30 de Septiembre, 2019

\section{Resumen}

En la fase definir de la metodología Lean Seis Sigma se deben identificar oportunidades de mejora, determinar e interpretar la voz del cliente para definir los objetivos con el equipo de trabajo que aplique las herramientas, habilidades y conocimientos para que la empresa sea productiva y competitiva. En el presente trabajo se muestra como obtener un objetivo que sea específico, realista, medible y a tiempo, siendo clave para una ejecución exitosa de mejora, el KPi que se trabajó fue el porcentaje de sacos con defecto en la primera sección de una maquiladora, identificando el promedio de sacos defectuosos, especificando bajar dicho promedio en un $90 \%$ para ser significativo y cuantificable, así la empresa pueda continuar con las fases de Medir, Analizar, Mejorar y Controlar, se muestran también dos de las operaciones críticas identificadas con los parámetros de calidad requeridos por el cliente así como el cálculo del Takt Time del proceso de la primera sección de la maquiladora con el fin de observar los cuellos de botella en las diferentes operaciones que conforman el área.

Lean Seis Sigma, Objetivo, Takt Time

\begin{abstract}
In the defining phase of the Lean Six Sigma Methodology, opportunities for improvement must be identified, determined and interpreted the client's voice to define the objectives with the work team that applies the tools, skills and knowledge so that the company is productive and competitive. This recent work shows how to obtain an objective that is specific, realistic, measurable and on time, being the key to a successful execution of improvement, the KPi that was worked was the percentage of bags with defect in the first section of a Manufacturer, identifying the average of defective bags, specifying to lower said average by $90 \%$ to be significant and quantifiable, so that the company can continue with the Measure, Analyze, Improve and Control phases, two of the critical operations identified with the parameters of quality required by the client as well as the calculation of the Takt Time of the process of the first section of the Manufacturer in order to observe the bottlenecks in the different operations that make up the area.
\end{abstract}

Lean Six Sigma, Objetive, Takt Time

Citación: HERNÁNDEZ-PASTRANA, Verónica Petra, KIDO-MIRANDA, Juan Carlos, PÉREZ-CABRERA, Pascual Felipe, y RODRÍGUEZ-BUCIO, Norma. Definición del KPi Porcentaje de sacos con defecto y Takt Time. Revista de Operaciones Tecnológicas. 2019. 3-11: 21-29

\footnotetext{
$\dagger$ Investigador contribuyendo como primer autor.
} 


\section{Introducción}

Lean Seis Sigma radica en descubrir en las empresas oportunidades de mejora, puesto que siempre existen desperdicios y variabilidad que pueden ser eliminados.

Uno de los principales despilfarros en las empresas es por defectos, significa una gran pérdida de productividad, debido a que incluye trabajos extra que deben realizarse para poder cumplir con el objetivo o meta establecida por día de producción y satisfacer al cliente con un producto o servicio de calidad.

El presente trabajo se desarrolla en una maquiladora con una producción diaria de 750 sacos para caballero al día, se presentan dos de las operaciones críticas de la primera sección, especificando los requerimientos de calidad que deben cumplir de acuerdo a lo que solicita el cliente.

Se establece el KPi porcentaje de sacos con defecto, con una métrica adecuada y aprobada por el equipo de trabajo, este objetivo fue mejorar el porcentaje de sacos con defecto en un $90 \%$.

Posteriormente se calculó el takt time, su importancia radica en que si se trabaja en la primera sección a un ritmo inferior de la demanda del cliente se tendrán que trabajar tiempos extra para satisfacer esta demanda, pero si la producción es mayor a lo demandado la línea de producción tendrá cuellos de botella como se muestra en este artículo. El takt time permitirá producir al ritmo que el cliente lo requiere.

Por último para conocer la capacidad de proceso (porcentaje de carga) se calcularon los tiempos ciclo de las 26 operaciones de la primera sección, y los tiempos caminar, dado la capacidad de proceso y la capacidad de producción se obtuvo el inventario por operación, reflejando los problemas de sobreproducción o falta de producción, es decir que no se producía al ritmo de lo que el cliente espera.

\section{Justificación}

Luis Socconini en su libro Lean Manufacturing menciona "Algunos expertos estiman que una empresa que tiene $10 \%$ de desperdicio, reduce hasta $40 \%$ su capacidad.
Es por eso que velocidad (Manufactura esbelta) y calidad (Seis Sigma) son caras de la misma moneda" [1].

En toda organización siempre será necesario aplicar la mejora, con la metodología Lean Seis Sigma se establecen objetivos que permitan a un grupo de trabajo identificar las metas a alcanzar.

En la maquiladora se sabe del porcentaje de sacos con defecto por lo que, es indispensable establecer un objetivo cuantitativo, alcanzable a corto plazo para reducir los retrabajos y pérdida de materiales, insumos, costos en mano de obra, costos por fallas internas y externas.

Es necesario contar con una meta clara, medible y bien planeada para alcanzar el éxito, si no se tiene existe una alta probabilidad de fracasar o tener grandes pérdidas.

Con productos defectuosos se pierden recursos empleados, ya que se invierten materiales, tiempo máquina, tiempo del recurso humano.

La empresa recibe pedidos de clientes con quienes se compromete a entregar en una fecha establecida su pedido, de ahí la importancia de conocer el Takt Time para cumplir con los tiempos de entrega.

\section{Contexto de la problemática}

La maquiladora se dedica a confeccionar saco y pantalón de caballero, cuenta con 30 años en el ramo de confección. Maquila sacos para marcas reconocidas. Laborando un total de 195 trabajadores, con horario de 10 horas al día de lunes a viernes.

Se ha detectado un porcentaje de sacos con defecto en la primera sección de la maquiladora.

La cantidad de sacos con defecto provoca que se eleven los costos de producción por retrabajo y trabajo de tiempos extra y de inspección de calidad, etc.

Los clientes han generado no conformidades, además de penalizar económicamente por la no entrega a tiempo, por lo que se tiene que buscar la causa raíz e implementar control de calidad más estricto continuando con la mejora del proceso. 


\section{Hipótesis}

La definición de un objetivo específico, realista y medible, así como el takt time y operaciones cuellos de botella son parámetros para establecer acciones a realizar en un proceso de mejora.

\section{Objetivos}

\section{Objetivo General}

Definir el objetivo cuantitativo a alcanzar y conocer el takt time y cuellos de botella en la primera sección de la maquiladora.

\section{Objetivos específicos}

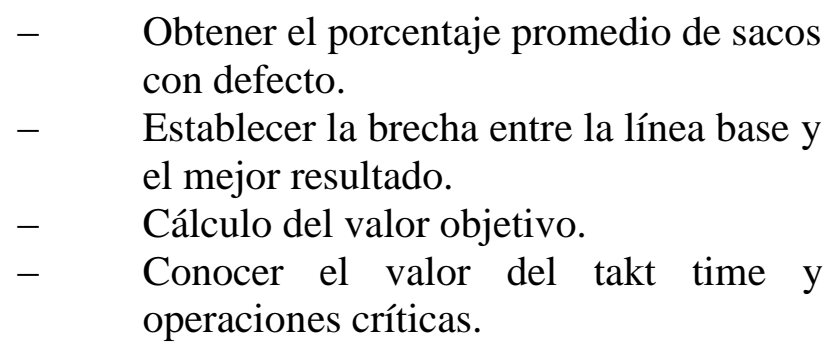

\section{Marco Teórico}

Manufactura Esbelta es el nombre que recibe el sistema Just In Time en occidente, también es llamada Manufactura de Clase Mundial y Sistema de Producción Toyota [1].

Manufactura esbelta también llamada producción ajustada la define Rajadell \& Sanchez [5] como "la persecución de una mejora del sistema de fabricación mediante la eliminación del desperdicio, entendiendo como desperdicio o despilfarro todas aquellas acciones que no aportan valor al producto y por las cuales el cliente no está dispuesto a pagar”.

Seis Sigma se enfoca principalmente a disminuir el desperdicio a través de la reducción de la variabilidad en los procesos.

La metodología de Six Sigma DMAIC (Define, Measure, Analyze, Improve, Control) se apoya en herramientas estadísticas y administrativas para mejorar, de manera tangible, los resultados de desempeño de los procesos y productos de una empresa [2]

Con Lean Seis Sigma se logra la reducción de variabilidad y desperdicio garantizando la calidad y productividad.
El uso del término SMART es atribuido a George T. Doran, quien en 1981 publicó un paper llamado "There's a S.M.A.R.T. Way to Write Management's Goals and Objectives”.

Un objetivo bajo el acrónimo SMART significa que debe ser Específico (Specific), Medible (Measurable), Alcanzable (Achievable), Relevante (Relevant) y a Tiempo (Time-bound)

La metodología Smart se basa en establecer metas que se puedan cumplir. De esta manera sabremos claramente cuando un objetivo ha sido alcanzado.

La medición del trabajo es esencial para valorar y planificar el trabajo productivo, para fijar plazos de entrega al cliente, equilibrar líneas de producción, asignar capacidades, tener una base objetiva para motivar a los trabajadores o medir su desempeño, establecer puntos de referencia con miras a la mejora (Arcusa, Gil \& Suñé) [6].

Socconini [1] define Tiempo Takt "velocidad a la que compra el cliente y es el tiempo al que el sistema de producción debe adaptarse para satisfacer las expectativas del cliente" y se obtiene dividiendo el tiempo total disponible entre la demanda del cliente.

Radajell Carreras Manuel en su libro "Lean Manufacturing. La evidencia de una necesidad" menciona que "producir según el takt time significa sincronizar el ritmo de la producción con el de las ventas de manera que se tiene una idea de la velocidad a la cual se debería estar produciendo"

\section{Metodología de Investigación}

Se inicia el trabajo recabando información del número de piezas con defecto que se obtenían al día en la primera sección, para conocer los porcentajes. Para fines prácticos se presenta una muestra de 12 días.

De lo anterior se obtiene la línea base, el mejor resultado, se calcula la brecha y se establece el objetivo.

En la primera sección de la maquiladora existen 26 operaciones de las cuales se muestran dos operaciones críticas con los parámetros de calidad que requiere el cliente. 
Posteriormente nos enfocamos a la obtención del takt time que es una herramienta de la metodología de Manufactura Esbelta diseñada para mejorar procesos en las empresas y con ello reducir los costos, aumentar la capacidad de producción para satisfacer al cliente. Para el cálculo de takt time se tomaron tiempos de las operaciones de la primera sección, entendiendo que la duración de un ciclo viene dada por el período entre la repetición del mismo evento que caracteriza el comienzo o el final de ese ciclo.

\section{Resultados}

\section{Operaciones críticas}

En la gráfica de Pareto (Gráfico 1) se muestran siete operaciones que se realizan en la primera sección de la maquiladora, siendo las más críticas Costados con un $29 \%$ y un $22.6 \%$ en la operación Vivos.

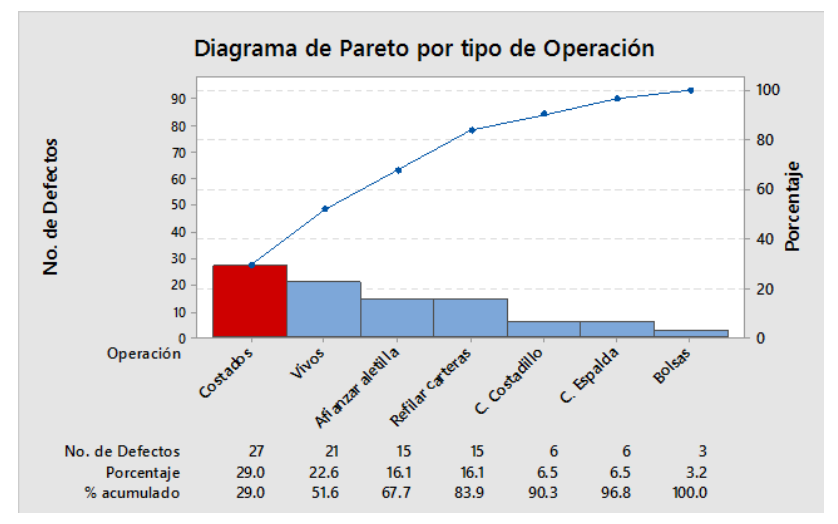

Gráfico 1 Diagrama de Pareto por operación

El esquema de la operación cerrar costados (Figura 1) muestra lo que espera el cliente, los requerimientos a cumplir de calidad son: el flojo sobre la espalda $2 \mathrm{~cm}$ debajo de la sisa hasta el aplomo del costado, el flojo de $5 \mathrm{~mm}$ debe estar bien distribuido, se debe respetar los márgenes y el ancho de la costura de $1.5 \mathrm{~cm}$ debe ser uniforme, respetar la continuidad de la sisa y la prenda debe estar libre de flojo a partir del aplomo.

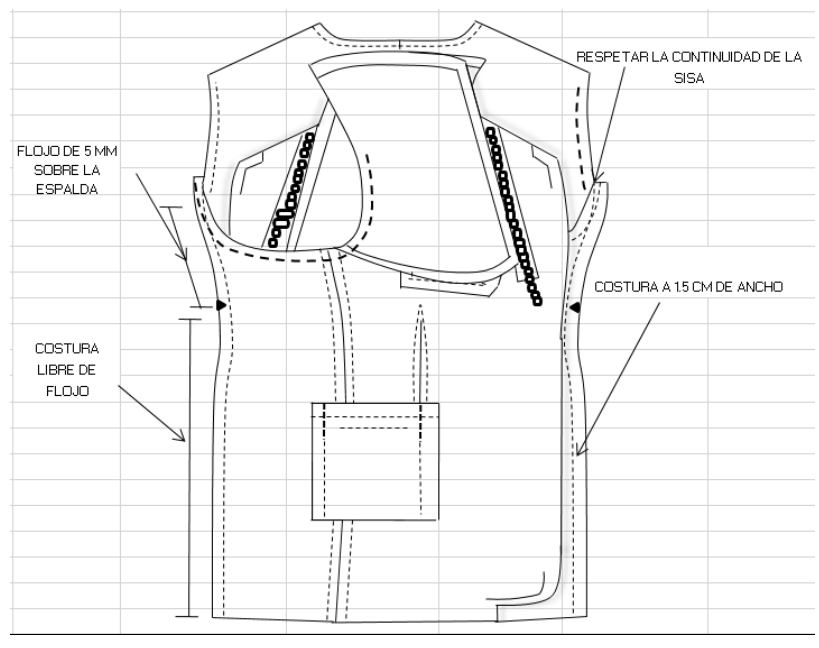

Figura 1 Esquema de operación cerrar costados

La operación plantar vivos debe cumplir los siguientes puntos de calidad, la costura debe iniciar y terminar exactamente en filo de los extremos de la cartera, la distancia entre la costura del vivo y la pinza de pecho debe ser de $1.5 \mathrm{~cm}$., el ancho de la cartera debe ser de $5.5 \mathrm{~cm}$ y $5 \mathrm{~mm}$ el ancho del vivo, cuidar que la cartera corresponda al delantero tanto en foleo como en el hilo derecho, el ancho de la cartera y vivo debe ser uniforme a lo largo de la costura, cuidar que la pinza posición bolsa quede centrada en la costura del vivo para que no se abra (Ver Figura 2).

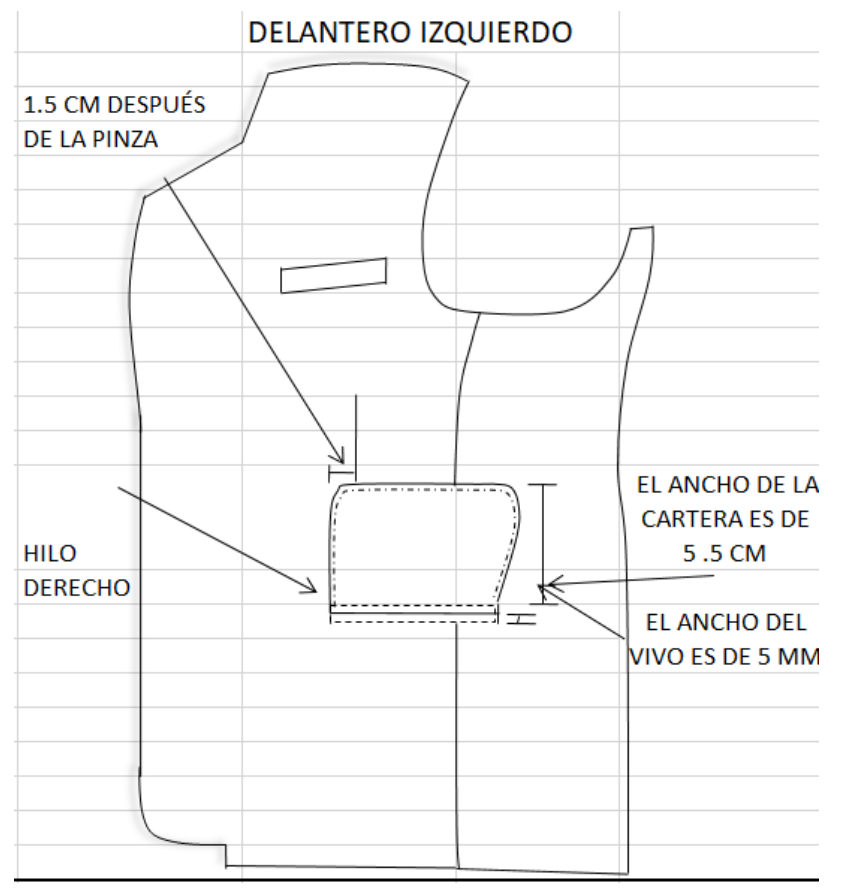

Figura 2 Esquema de operación Plantar vivos 


\section{Cálculo del KPi}

La primera fase de la metodología Lean Seis Sigma es Definir, un punto importante es determinar la línea base del desempeño actual para el cual se desea un nivel de desempeño, con ello se puede obtener una idea de la magnitud del problema o área de mejora, esta línea base siempre se debe expresar en un nivel de unidades, para nuestro proyecto fue el porcentaje de sacos con defecto. En la definición de nuestro objetivo el métrico es el porcentaje de sacos con defecto, la unidad de medida es el número de sacos defectuosos, el método de cálculo es piezas de sacos con defecto diarias entre piezas producidas diarias y la dirección de mejora es menor es mejor.(Tabla 1)

\begin{tabular}{|c|c|c|c|}
\hline Métrico & $\begin{array}{l}\text { Unidad de } \\
\text { Medida }\end{array}$ & $\begin{array}{l}\text { Método de } \\
\text { cálculo }\end{array}$ & $\begin{array}{l}\text { Dirección } \\
\text { de mejora }\end{array}$ \\
\hline $\begin{array}{l}\text { Producción } \\
\text { de sacos } \\
\text { con defecto }\end{array}$ & $\begin{array}{l}\text { Número de } \\
\text { Sacos } \\
\text { defectuosos }\end{array}$ & $\begin{array}{l}\text { Piezas de } \\
\text { sacos con } \\
\text { defecto } \\
\text { diarias } \\
\text { entre } \\
\text { piezas } \\
\text { produci- } \\
\text { das diarias. }\end{array}$ & $\begin{array}{l}\text { Menor es } \\
\text { mejor }\end{array}$ \\
\hline
\end{tabular}

Tabla 1 Datos para definir el objetivo

En la tabla 2 de acuerdo a los reportes de control de producción de la Tercera Sección, se muestran los valores del total de piezas producidas en doce días con el total de piezas con defecto, de los cuales se obtiene el porcentaje de sacos con defecto para cada día, resultando un promedio de $1.07 \%$ de sacos con defecto en la primera sección de la maquiladora, del 17 de septiembre 2018 hasta el 28 de septiembre del 2018 (como primera muestra, que es la que se presenta en el presente documento).

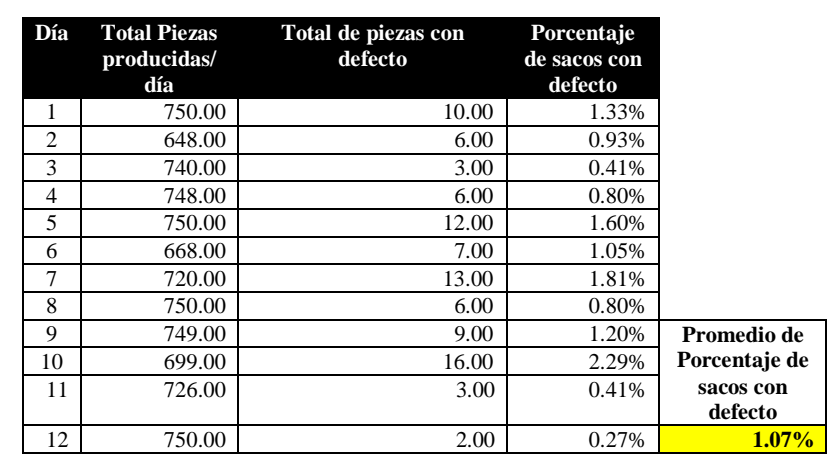

Tabla 2 Porcentaje de sacos con defecto
Los cálculos para definir el valor de nuestro objetivo a alcanzar con la mejora es el siguiente:

La línea base (LB) es el promedio que se obtiene del porcentaje de sacos con defecto, siendo este de $1.07 \%$.

El mejor resultado (MR), es el menor porcentaje de sacos con defecto igual a $0.27 \%$.

La Brecha es igual a la diferencia entre el Promedio (LB) y el Mejor Resultado (MR) dando un total de $0.8 \%$.

Para obtener el valor del objetivo a alcanzar después de la mejora es: a la línea base le restamos el valor calculado de multiplicar 0.9 por la brecha. El 0.9 representa que queremos alcanzar a mejorar el $90 \%$ de los sacos, el valor del objetivo es $0.35 \%$.

Cabe señalar que es recomendable establecer un $70 \%$ en lugar de un $90 \%$ para que sea una meta a alcanzar a corto plazo, y el personal no se vea tan estresado para alcanzar la misma. Se decidió por el $90 \%$, porque el porcentaje de sacos con defecto era poco en relación con otras áreas detectadas de la maquiladora, que son oportunidades que se presentarán en otro documento.

Con el fin de visualizar con claridad los datos y objetivo nominal a alcanzar se realizó el gráfico 2, donde apreciamos cada uno de los porcentajes obtenidos, la línea base y el objetivo a alcanzar de $0.35 \%$.

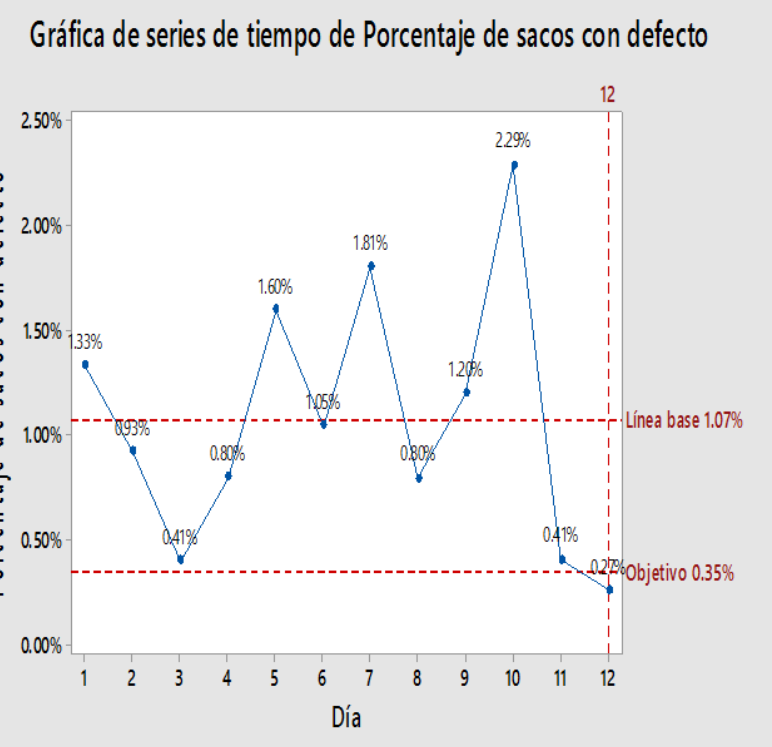

Gráfico 2 Serie de tiempo de porcentaje de sacos con defecto y objetivo

HERNÁNDEZ-PASTRANA, Verónica Petra, KIDO-MIRANDA, Juan Carlos, PÉREZ-CABRERA, Pascual Felipe, y RODRÍGUEZ-BUCIO, Norma. Definición del KPi Porcentaje de sacos con defecto y Takt Time. Revista de Operaciones Tecnológicas. 2019 


\section{Cálculo del Takt Time}

Takt Time es una palabra alemán que significa ritmo, para su cálculo necesitamos los datos de pedidos de los clientes, es decir, la demanda que tiene la maquiladora, el Takt Time debe marcar el ritmo de la línea de producción, por lo que podemos decir que también afecta el flujo de la cantidad de operarios, frecuencia de alimentación de la línea, número de componentes de los proveedores consumidos.

Los datos recabados para el cálculo del Takt Time se detallan a continuación:

La empresa labora 10 horas al día, desde las 7:00 a las 17:00 hrs., por lo que el tiempo pagado es de 36000 segundos. Cabe señalar que los paros programados varía en cada empresa, en nuestro caso sólo consideramos el horario de comida de 30 minutos, mantenimiento autónomo de 10 minutos como el cambio de una aguja o limpieza de la máquina, entre otros, junta de arranque de 5 minutos sólo para dar indicaciones necesarias y 15 minutos de tiempo para realizar sus necesidades fisiológicas o tomar agua.

Tiempo diario total disponible para producción $=$ tiempo de trabajo - paradas programadas

En una línea de producción, cada intervalo definido por takt-time, una unidad debe terminarse.

Entonces en el área de producción de la primera sección, se tienen:

36000 segundos de tiempo pagado,

$32,400 \mathrm{seg}$. de tiempo diario total disponible

3600 seg paros programados

El cálculo del takt time es dividir el tiempo diario total disponible de 32,400 segundos entre 750 sacos diarios de producción.

Resultado del Takt Time de 43 segundos, es decir, cada en $43 \mathrm{seg}$. se debe terminar una pieza en la primera sección como se muestra en la tabla 3. Este tiempo varía de acuerdo al modelo del saco, ya que se tomó el tiempo del saco más sencillo a realizar. Existen sacos donde la tela es de cuadros, o la tela es poco manejable lo que implica tener mayor cuidado en el trabajo a realizar así como una supervisión más controlada para evitar alto porcentaje de sacos con defecto.

\begin{tabular}{|c|c|c|c|}
\hline Descripción & Turno & Total & \\
\hline Horarios & $7: 00$ a $17: 00$ & & \\
\hline $\begin{array}{l}\text { Tiempo pagado } \\
\text { (segs) }\end{array}$ & 36000 & 36,000 & \\
\hline \multicolumn{4}{|l|}{$\begin{array}{l}\text { Paros } \\
\text { programados: }\end{array}$} \\
\hline Comida & 1800 & 1800 & \\
\hline $\begin{array}{l}\text { Mantenimiento } \\
\text { autónomo }\end{array}$ & 600 & 600 & \\
\hline Junta de arranque & 300 & 300 & \\
\hline Descanso & 900 & 900 & \\
\hline $\begin{array}{l}\text { Total de paros } \\
\text { programados }\end{array}$ & 3600 & (A ) 3,600 & \\
\hline $\begin{array}{l}\text { Tiempo diario } \\
\text { total disponible }\end{array}$ & 32,400 & ( В ) 32,400 & seg/dia \\
\hline & & (C) 750 & $\begin{array}{l}\text { pzs / } \\
\text { dia }\end{array}$ \\
\hline & & $\begin{array}{l}\text { Takt Time }= \\
43\end{array}$ & $\begin{array}{l}\text { seg } \\
/ \mathrm{pza}\end{array}$ \\
\hline
\end{tabular}

Tabla 3 Cálculo de Takt Time

\section{Tiempo ciclo}

El tiempo de ciclo individual es el tiempo que dura cada operación individual. Se tomó el tiempo considerando a un operario capacitado, que realice y conozca bien su actividad siguiendo el método preestablecido.

En la tabla 4 se muestra el tiempo ciclo de 26 operaciones de la primera sección de la maquiladora.

Por ejemplo el tiempo ciclo para la operación coser pinza es de 25.2 segundos.

En el enfoque cliente-proveedor, el proveedor busca un desempeño en tiempo ciclo, costo y defectos para cumplir las expectativas de los clientes en entrega, precio y calidad. 


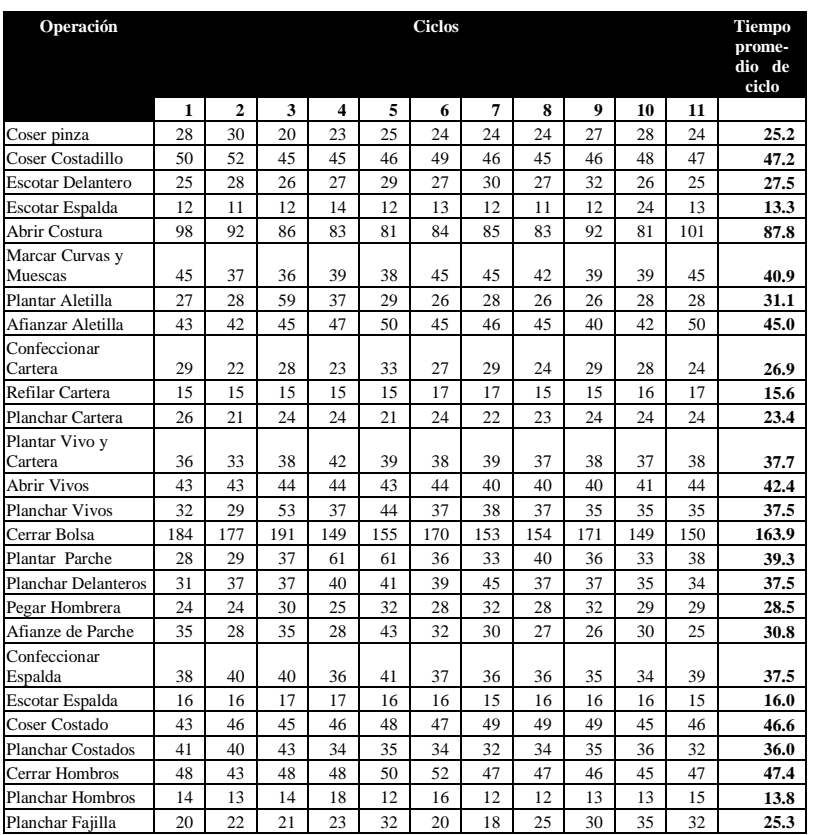

Tabla 4 Tiempo ciclo por operación

\section{Cálculo de la Capacidad de proceso (\% de carga)}

Los datos del tiempo manual son los valores que están en la tabla 5 como tiempo ciclo. El tiempo caminar son los segundos de pasar la pieza a otra operación y el tamaño del lote o pieza en nuestro caso es un saco, con estos datos se calcula el caminar por pieza que se muestra en la última columna de la tabla 6 .

\begin{tabular}{|l|r|}
\hline \multicolumn{1}{|c|}{ Sección } & $\begin{array}{c}\text { Segunda } \\
\text { Tiempo en } \\
\text { segundos }\end{array}$ \\
\hline Nombre de la Operación & $\begin{array}{r}\text { Tiempo Manual } \\
\text { C }\end{array}$ \\
\hline Coser pinza & $\mathbf{2 5 . 2}$ \\
\hline Coser Costadillo & $\mathbf{4 7 . 2}$ \\
\hline Escotar Delantero & $\mathbf{2 7 . 5}$ \\
\hline Escotar Espalda & $\mathbf{1 3 . 3}$ \\
\hline Abrir Costura & $\mathbf{8 7 . 8}$ \\
\hline Marcar Curvas y Muescas & $\mathbf{4 0 . 9}$ \\
\hline Plantar Aletilla & $\mathbf{3 1 . 1}$ \\
\hline Afianzar Aletilla & $\mathbf{4 5 . 0}$ \\
\hline Confeccionar Cartera & $\mathbf{2 6 . 9}$ \\
\hline Refilar Cartera & $\mathbf{1 5 . 6}$ \\
\hline Planchar Cartera & $\mathbf{2 3 . 4}$ \\
\hline Plantar Vivo y Cartera & $\mathbf{3 7 . 7}$ \\
\hline Abrir Vivos & $\mathbf{4 2 . 4}$ \\
\hline Planchar Vivos & $\mathbf{3 7 . 5}$ \\
\hline Cerrar Bolsa & $\mathbf{1 6 3 . 9}$ \\
\hline Plantar Parche & $\mathbf{3 9 . 3}$ \\
\hline Planchar Delanteros & $\mathbf{3 7 . 5}$ \\
\hline Pegar Hombrera & $\mathbf{2 8 . 5}$ \\
\hline Afianze de Parche & $\mathbf{3 0 . 8}$ \\
\hline Confeccionar Espalda & $\mathbf{3 7 . 5}$ \\
\hline Escotar Espalda & $\mathbf{1 6 . 0}$ \\
\hline Coser Costado & $\mathbf{4 6 . 6}$ \\
\hline Planchar Costados & $\mathbf{3 6 . 0}$ \\
\hline Cerrar Hombros & $\mathbf{4 7 . 4}$ \\
\hline Planchar Hombros & $\mathbf{1 3 . 8}$ \\
\hline Planchar Fajilla & $\mathbf{2 5 . 3}$ \\
\hline
\end{tabular}

Tabla 5 Tiempo de ciclo

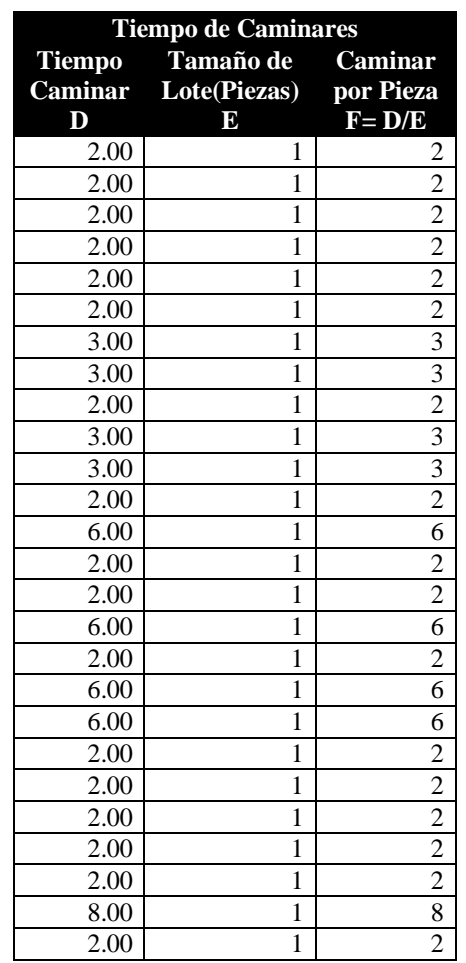

Tabla 6 Tiempo caminar

En la tabla 7 para el tiempo total por pieza debemos sumar el tiempo manual y el caminar por pieza, seguido del cálculo capacidad de producción (piezas diarias, dividiendo el tiempo disponible de 32,400 segundos entre el tiempo total por pieza, por último la capacidad de proceso o porcentaje de carga se obtiene dividiendo la demanda diaria de 750 sacos entre la capacidad de producción. Lo señalado en rojo son las operaciones que están provocando cuellos de botella por no ajustarse al takt time.

\begin{tabular}{|c|c|c|}
\hline \multicolumn{3}{|c|}{ Resumen en segundos } \\
\hline $\begin{array}{c}\text { Tiempo Total } \\
\text { por Pieza } \\
\mathrm{J}=\mathrm{C}+\mathrm{F}\end{array}$ & $\begin{array}{l}\text { Capacidad de } \\
\text { producción } \\
\text { (pzs diarias) } \\
\text { M=K / J }\end{array}$ & $\begin{array}{c}\text { Capacidad de } \\
\text { proceso } \\
\text { (\% de carga) } \\
\text { L / M }\end{array}$ \\
\hline 27.18 & 1191.97 & $63 \%$ \\
\hline 49.18 & 658.78 & $114 \%$ \\
\hline 29.45 & 1100.00 & $68 \%$ \\
\hline 15.27 & 2121.43 & $35 \%$ \\
\hline 89.82 & 360.73 & $208 \%$ \\
\hline 42.91 & 755.08 & $99 \%$ \\
\hline 34.09 & 950.40 & $79 \%$ \\
\hline 48.00 & 675.00 & $111 \%$ \\
\hline 28.91 & 1120.75 & $67 \%$ \\
\hline 18.64 & 1738.54 & $43 \%$ \\
\hline 26.36 & 1228.97 & $61 \%$ \\
\hline 39.73 & 815.56 & $92 \%$ \\
\hline 48.36 & 669.92 & $112 \%$ \\
\hline 39.45 & 821.20 & $91 \%$ \\
\hline 165.91 & 195.29 & $384 \%$ \\
\hline 45.27 & 715.66 & $105 \%$ \\
\hline 39.55 & 819.31 & $92 \%$ \\
\hline 34.45 & 940.37 & $80 \%$ \\
\hline 36.82 & 880.00 & $85 \%$ \\
\hline 39.45 & 821.20 & $91 \%$ \\
\hline 18.00 & 1800.00 & $42 \%$ \\
\hline 48.64 & 666.17 & $113 \%$ \\
\hline 38.00 & 852.63 & $88 \%$ \\
\hline 49.36 & 656.35 & $114 \%$ \\
\hline 21.82 & 1485.00 & $51 \%$ \\
\hline 27.27 & 1188.00 & $63 \%$ \\
\hline
\end{tabular}

Tabla 7 Capacidad de Proceso

HERNÁNDEZ-PASTRANA, Verónica Petra, KIDO-MIRANDA, Juan Carlos, PÉREZ-CABRERA, Pascual Felipe, y RODRÍGUEZ-BUCIO, Norma. Definición del KPi Porcentaje de sacos con defecto y Takt Time. Revista de Operaciones Tecnológicas. 2019 
Tomando como ejemplo la operación Coser Costadillo se obtuvo una capacidad de proceso de $114 \%$ (Tabla 7) resultado que se explica porque la capacidad de producción o piezas diarias producidas es de 658.78 y la demanda diaria que se tiene que cumplir es de 750 sacos; por lo que el inventario en esta operación implica un número de menos 91.22 (Tabla 8). De la misma forma la operación Cerrar bolsa tiene una capacidad de proceso de $384 \%$, debido a que la capacidad de producción o piezas diarias producidas es de 195.29 resultando un inventario de menos 554.71 piezas.

Caso contrario la operación Escotar Espalda con un $42 \%$ de capacidad de proceso ya que su capacidad de producción es de 1800 piezas, valor que es mucho mayor que la demanda diaria por lo que refleja un inventario de 1050 piezas. El que exista inventario de piezas en exceso o deficientes será siempre un problema que hay que atender. Este problema genera que existan operarios con sobrecarga o con poca carga de trabajo, requiriendo que se realice un balanceo en las operaciones de la línea. Con la aplicación de la metodología Lean Seis Sigma se puede disminuir la variabilidad y las mudas de sobreproducción, sobreinventario, de productos defectuosos, de espera $y$ movimientos innecesarios del trabajador, que están presentes debido a los resultados mostrados anteriormente.

\begin{tabular}{|l|r|}
\multicolumn{1}{|c}{$\begin{array}{c}\text { Nombre de la } \\
\text { Operación }\end{array}$} & $\begin{array}{c}\text { Inventario } \\
\text { diario en piezas } \\
\text { M-L }\end{array}$ \\
\hline Coser pinza & 441.97 \\
\hline Coser Costadillo & -91.22 \\
\hline Escotar Delantero & 350.00 \\
\hline Escotar Espalda & 1371.43 \\
\hline Abrir Costura & -389.27 \\
\hline Marcar Curvas y & 5.08 \\
Muescas & 200.40 \\
\hline Plantar Aletilla & -75.00 \\
\hline Afianzar Aletilla & 370.75 \\
\hline Confeccionar Cartera & 988.54 \\
\hline Refilar Cartera & 478.97 \\
\hline Planchar Cartera & 65.56 \\
\hline Plantar Vivo y Cartera & -80.08 \\
\hline Abrir Vivos & 71.20 \\
\hline Planchar Vivos & -554.71 \\
\hline Cerrar Bolsa & -34.34 \\
\hline Plantar Parche & 69.31 \\
\hline Planchar Delanteros & 190.37 \\
\hline Pegar Hombrera & 130.00 \\
\hline Afianze de Parche & 71.20 \\
\hline Confeccionar Espalda & 1050.00 \\
\hline Escotar Espalda & -83.83 \\
\hline Coser Costado & 102.63 \\
\hline Planchar Costados & -93.65 \\
\hline Cerrar Hombros & 735.00 \\
\hline Planchar Hombros & 438.00 \\
\hline Planchar Fajilla & \\
\hline
\end{tabular}

Tabla 8 Inventario diario de piezas
En la gráfica 3 se muestran las operaciones de la primera sección con el tiempo ciclo para cada una de ellas, la línea punteada es el takt time obtenido de 43 segundos, la línea punteada tiene el objetivo de visualizar las operaciones cuellos de botella como son coser costadillo, abrir costura, marcar curvas y muescas, afianzar aletilla, abrir vivos, cerrar bolsa y plantar parche. En estas operaciones es necesario realizar balanceo de líneas para equilibrar los inventarios mostrados anteriormente y mejorar la capacidad de producción además de implementar mejoras en todo el proceso que disminuya la variabilidad en cada una de las piezas y elevar la calidad.

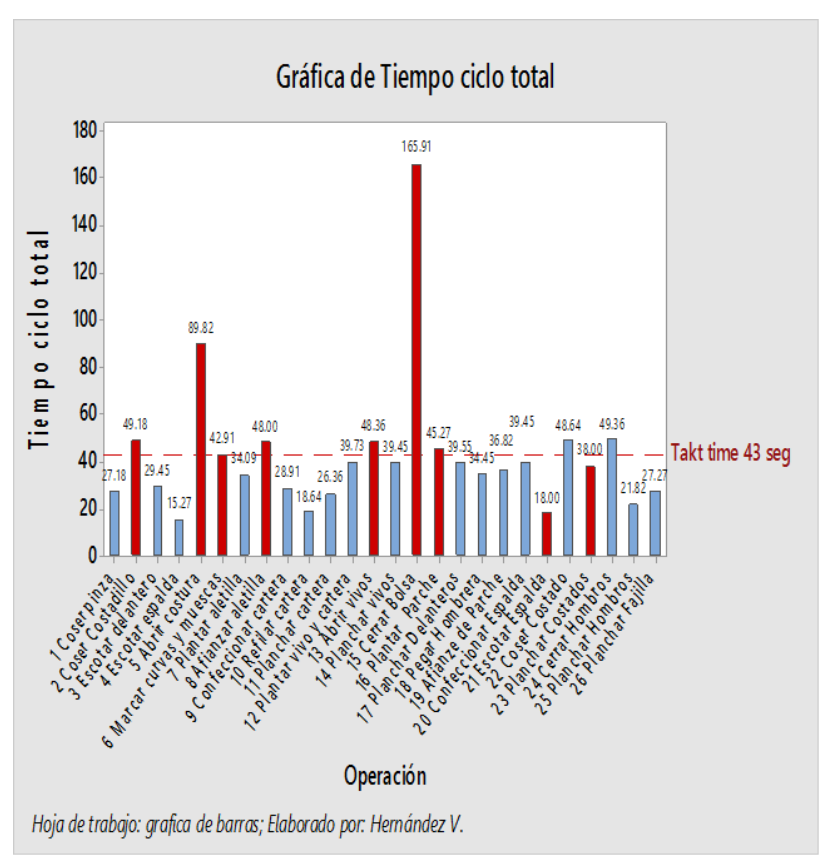

Gráfico 3 Tiempo ciclo por operación y takt time

Se puede observar además en la gráfica 3 que no todas las operaciones necesitan el mismo tiempo, existen operarios que realizan su actividad en menor tiempo o mayor tiempo que el takt time, por lo que se tiene que equilibrar la línea, proceso mediante el cual se distribuyen niveladamente los elementos del trabajo dentro de un flujo de valor con el fin de satisfacer el takt time.

\section{Conclusiones}

Luis Socconini expresa que "La variación alrededor de un objetivo afecta al costo principalmente a través de:

Las fluctuaciones alrededor del objetivo deseado incrementan el costo de la operación existente. 
- Las fluctuaciones alrededor del objetivo deseado incrementan el costo de las operaciones posteriores del proceso.

- Amplifica los procesos en términos de tolerancias, recursos, materias primas y número de unidades iniciadas.

Disminuye la eficiencia debido a la necesidad de incrementar la complejidad del proceso".

La importancia de establecer un objetivo SMART radica en los puntos anteriores, el establecido en nuestro trabajo es de $0.35 \%$, lo que significa bajar a este porcentaje el número de sacos con defecto.

Cuando se utilizan objetivos SMART y metas claras, el desempeño y el compromiso del personal mejoran significativamente.

Rajadell, M \& Sanchez, J. L expresan que los beneficios del Takt Time se reflejan en aspectos como:

- Un ritmo estable de producción nivelada.

- No hay exceso de producción.

- Un flujo de componentes estable y nivelado.

- Un número correcto de operarios en cada proceso.

- Una mayor capacidad para planificar otras actividades en la producción.

El tiempo Takt es una de las mediciones importantes para elaborar el mapa de valor que se utilizan para conocer el proceso a fondo tanto dentro de la maquiladora como en la cadena de suministro y diseñar un sistema que se adapte a las fluctuaciones de la demanda de los clientes.

Con la detección de los cuellos de botella se enfocarán los esfuerzos en las operaciones críticas y se tendrá que realizar un balanceo de la línea. Con el tiempo ciclo y el Takt Time se puede calcular el número de operarios en cada estación de trabajo.

El implementar la metodología Lean Seis Sigma puede tener los siguientes beneficios en la maquiladora

\footnotetext{
- $\quad$ Reducción de scrap

- Reducción en tiempos de entrega y costos

- Aplicar la metodología a otras secciones de la planta con el mismo problema
}
- Aprendizaje para poder llevar a cabo proyectos Lean Six Sigma de mayor complejidad
- Reducción de No Conformidades por parte del cliente.
- $\quad$ Reducción de sacos con defecto.

\section{Agradecimiento}

En primer lugar agradecemos a la maquiladora por brindarnos la oportunidad de realizar el proyecto, adquiriendo experiencia en el ramo.

Al Tecnológico Nacional de México/Instituto Tecnológico de Iguala por brindarnos las facilidades para poder asistir a la maquiladora como parte de nuestra capacitación como docentes e investigadores.

\section{Referencias}

[1] Socconini, L. (2009) Lean Manufacturing paso a paso. México. Grupo editorial Norma

[2] Socconini, L. (2016). Lean Six Sigma Green Belt. México. Alfaomega.

[3] Imai, M. (2009) Kaizen. México. Grupo Editorial Patria.

[4] Reis, R. \& Valle, J. (2001). Takt-time: conceptos y contextualización dentro del sistema de producción Toyota. Gestión y Producción 8 (1), 1-18 https://dx.doi.org/10.1590/S0104530X2001000100002

[5] Rajadell, M \& Sanchez, J. L. Lean manufacturing. La evidencia de una necesidad. Recuperado de https://books.google.es/books?hl=es\&lr=\&id=1 R2xgsdmdUoC\&oi=fnd\&pg=PR1\&dq=related: KvvVrud4f2AJ:scholar.google.com/\&ots $=\mathrm{K} 7 \mathrm{O}$ pFa6gBZ\&sig=cjDxpOFNtZt0k52tZxot0nWC

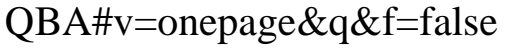

[6] Arcuza, I., Gil, F. \& Suñé, A. Manual Práctico de Diseño de Sistemas Productivos. Recuperado de https://books.google.es/books?hl=es\&lr=\&id= AkR_hCGsTIUC\&oi=fnd\&pg=PR13\&dq=tiem po+

ciclo+por+los+operarios \&ots $=\mathrm{aH} \mathrm{xCyZN7Pl \& s}$ ig=-QpsDgbC8HGV0-

n8xZafs9JsORY\#v=onepage \&q= tiempo\%20ciclo\%20por\%20los\%20operarios\& $\mathrm{f}=$ false 